\title{
Dynamical Modelling of the Process of Separation and Sieving of Wet Land Mass by Oscillatory Transporting Platform
}

\author{
Goran Mihajlović1* ${ }^{*}$ Milomir Gašić2 ${ }^{2}$ Mile Savković ${ }^{3}$ \\ ${ }^{1}$ Visoka tehnička mašinska škola strukovnih studija Trstenik \\ ${ }^{2}$ Fakultet za mašinstvo i građevinarstvo u Kraljevu Univerziteta u Kragujevcu
}

This paper presents an adequate mathematical model and real-time dynamic analysis of separation and sieving of wet land mass by using oscillatory transporting platform. The platforms can be used in various branches and fields of technology (agriculture, mining, construction, and the like). Regardless of the application area, the principle of platform operation (in terms of separation and sieving of wet land mass) is the same for all cases of its application, which makes it possible to generalize the obtained theoretical and practical research results. In this way, the results would become relevant and due to their universal character they would have a great applicative value in the design of similar machines or even new machines and technological systems that use the same type of platform.

Keywords: oscillatory transporting platform, wet land mass, separation, sieving, mathematical model, stability of the platform, reserve of stability of the platform.

\section{INTRODUCTION}

Separation (segmentation) is a physical or chemical process in which some less important components (called tailings) are removed, while the material itself is enriched with more valuable ingredients in order to be efficiently used both in technical and economical terms [3].Separation process of some ingredients is always preceded by the processes of breaking and grinding of solid mass, which are usually performed on machines called crushers (coarse and medium grinding) and mills (fine grinding). The level of crushing is the relationship between grain size after and before crushing.In practice, the most frequent types of separation are [3]:

- mechanical separation always involves prefragmenting and sieving of the mass, and then its later enrichment using different physical and chemical properties of individual component ingredients (e.g. different specific weights, i.e. densities, different settling rates, etc.);

- magnetic separation can be applied to materials with different magnetic properties, allowing the individual components to be separated in strong magnetic fields (for example, ferromagnetic and paramagnetic ores, Fig. 1);

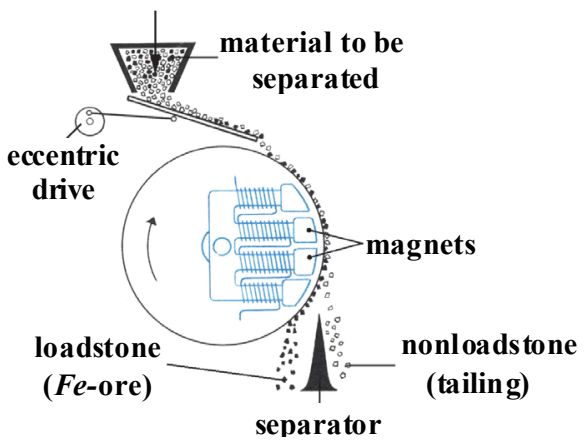

Fig. 1. Magnetic separator
- flotation separation is based on differences related to surface properties of materials, which in terms of water absorption can be hydrophilic (they absorb water and fall to the bottom of so called flotation cell) or hydrophobic (they do not absorb water and rise to the surface of flotation cell). Separated materials are then collected by specially developed techniques and procedures (Fig. 2).

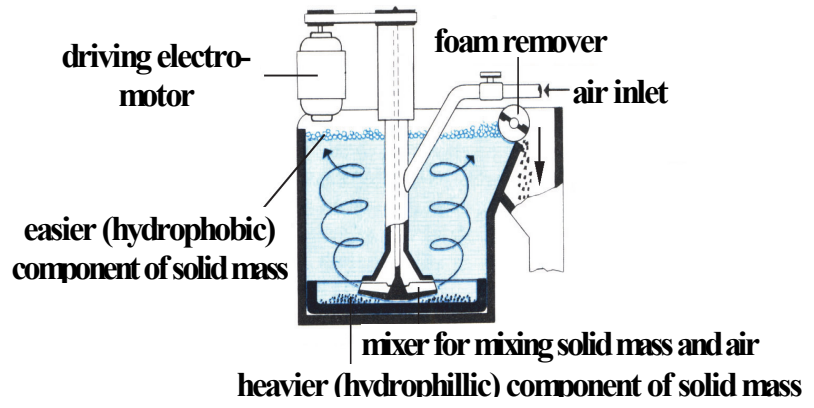

Fig. 2. Flotation separator (flotation cell)

However, it has been discovered recently that the mentioned processes of breaking and separating solid mass, besides the already mentioned methods, can also be performed in a very successful and rational manner by using special VIBRATING OSCILLATORY TRANSPORTATION PLATFORMS (Fig. 3). The operation of these platforms is normally influenced by many different factors, and since it is a relatively new and unknown issue, it is necessary to carry out extensive theoretical and practical research in order to obtain relevant results necessary for a complete understanding of the processes mentioned above. The aim is, as always in similar situations, to use the obtained data and drawn conclusions for the design, construction and exploitation of real mechanical and technological systems 
which have these oscillatory platforms installed as the working elements [7].

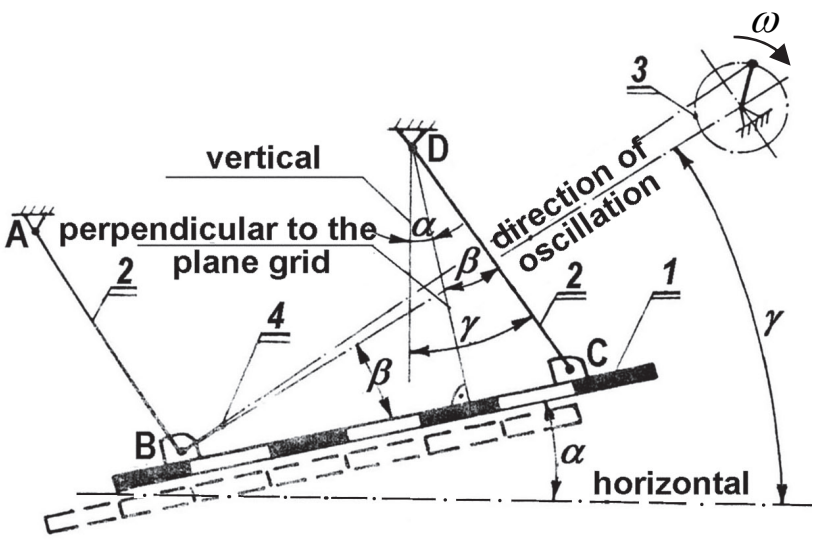

Fig. 3. General scheme of oscillatory transporting platform

(1-platform; 2-suspension; 3-excenter; 4-driving lever; A-amplitude of the platform oscillation (radius of the excenter); $\varphi$-phase of platform oscillation; $\alpha, \beta, \gamma$-influential angles of the platform; $A, B, C, D$-supporting points of the platform)

\section{MODEL OF THE SEPARATION AND SIEVING OF WET LAND MASS ON OSCILLATORY TRANSPORT PLATFORM}

Figure 4 shows a schematic and graphical representation of the transport platform, whereby the wet land mass is sieved through its grid. The following parameters are analyzed [2]:

$$
\begin{aligned}
& Q_{1}\left[\frac{m^{3}}{s}\right] \text { - amount of wet land mass delivered on the } \\
& \text { platform; }
\end{aligned}
$$

$P\left[m^{2}\right]$ - the total area of the platform grid through which the wet land mass is being sieved, where:

$$
P=P_{1}+P_{2}+\ldots+P_{n}=\sum_{i=1}^{n} P_{i}=\sum_{i=1}^{n}(l \cdot d)=\prod_{i=1}^{n}\left(n \cdot P_{i}\right)
$$

$P_{i}\left[m^{2}\right]$ - elementary surface of one $\left(i^{\text {th }}\right)$ area of platform grid through which wet land mass is sieved (fig. 4a);

$l[m]$ - length of platform grid through which wet land mass is sieved (fig. 4a);

$d[m]$ - size of the hole (perforation) on the platform grid (fig. 4a);

$n$ - the total number of elementary areas on the platform grid (fig. 4a).

If dry and friable land mass is delivered to the platform grid (the optimal conditions for transport and separation of solid mass), then the effect of separation and sieving is maximal and the fineness coefficient of separation is close to one $\left(\eta \approx 1\right.$, i.e. $\left.Q_{1} \approx Q_{2}\right)$. Mass sieving begins practically at the same moment when the solid mass reaches the grid of the platform (there is no "delay" time). (a)
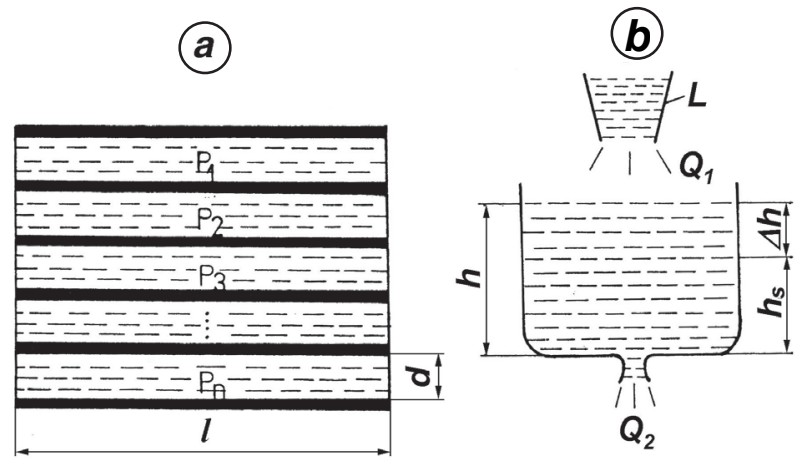

Fig. 4. Schematic presentation of the separation of wet land mass by using oscillatory transporting platform

However, if wet and plastic land mass (the most unfavorable conditions for transport and separation of solid mass) is delivered onto the grid of the platform, the fineness coefficient of separation rapidly decreases and in extreme cases tends to zero $(\eta \rightarrow 0)$, which can cause a complete loss of working effect of platform machine. Then a new parameter that expresses the amount of non-sieved wet land $Q^{*}\left[\mathrm{~m}^{3} / \mathrm{s}\right]$ can be defined:

$$
Q^{*}=Q_{1}-Q_{2}
$$

It is particularly important to note that the sieving of wet land mass does not start at the very moment when it is delivered on the grid of the platform, but after a certain time $t$ which is called a delay time of the output parameter $\left(Q_{2}\right)$ relative to the input parameter $\left(Q_{1}\right)$. The reason for this delay is sticking of wet land mass to the platform grid due to the strong cohesion and adhesion forces that contribute to forming a specific physical and chemical joint between the land particles and the grid, which is difficult to disassemble. Time delay $\tau$ is used as a measure of stickiness which in mathematical model can be conditionally understood as a value which is analogous to "stickiness coefficient" expressing the intensity of said stickiness. Then a basic rule is applied: the longer delay time, the higher the stickiness intensity. If wet land mass is delivered on the platform through a funnel $(L)$ (fig. $4 \mathrm{~b})$, the analysis of the sieving process can start from the moment $\boldsymbol{t}=\boldsymbol{t}_{\boldsymbol{t}}$ at which the balanced state (i.e. steady state) is established according to equation (2), which corresponds to point $S$ in the diagram of sieving (fig. 5c).

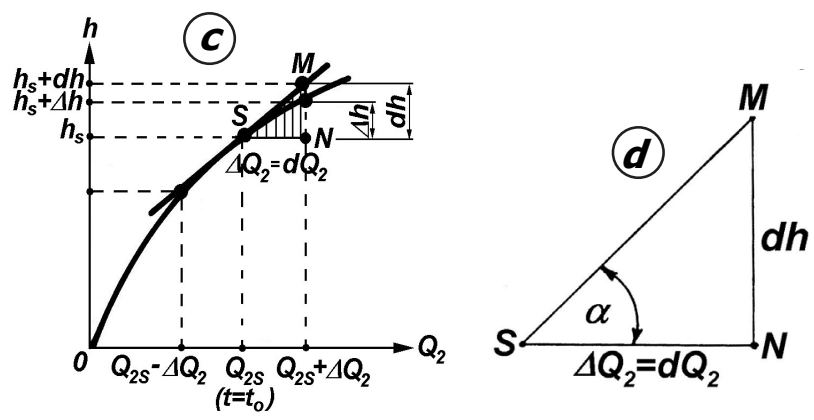

Fig. 5. Graphical presentation of the process of separation of wet land mass by using oscillatory transporting platform

Supposing that the supply of wet land on the platform is suddenly increased by a value $\Delta Q_{l}$, then the height of land 
on the platform is also increased by a value $\Delta h$, and thus the amount of sieved wet land mass will be increased by the value $\Delta Q_{2}$ (fig. 4.b). Increased level of wet land mass on a platform per unit of time is:

$$
\frac{\Delta h}{\Delta t}=\frac{\Delta Q_{1}-(1-K \tau) \Delta Q_{2}}{P}\left[\frac{m}{s}\right]
$$

$K_{\tau}=[0 \ldots 1]$ - delay coefficient of sieved land mass relative to the delivered land mass, which has extreme values in the following cases:

$K_{\tau}=0$ - for entirely dry and friable land mass ( $\eta \approx 1$, i.e. $Q_{1} \approx Q_{2}$, almost all delivered land mass would be fully sieved, so the delay time would be $\tau \approx 0$ );

$K_{\tau}=1$ - for maximally wet and plastic land mass ( $\eta=0$, this would make the platform stop, i.e. the sieving would be stopped because the land mass sticks to the grid, where the delay time approaches infinity, $\tau \rightarrow \propto$ ).

Unlike dry and friable land mass where diagram of sieving is presented by a straight line (linear function, [1]), sieving of wet and plastic land mass has a nonlinear character and therefore is presented by a curve [2]. Thus in this case it is necessary to apply the process of linearization, i.e. approximation of a portion of function by a straight line (first-order polynomial). In theory of mathematical modeling, the most common case is to approximate a part of the function $f(x)$ by tangent near a characteristic point on the diagram. In our case that is a point $S\left[Q_{2} ; h_{S}\right]$ which has already been stated to represent the equilibrium state of the system.

If the increased level of wet land mass on the platform $\Delta h$ is written by means of differential:

$$
d(\Delta h)=\frac{1}{P}\left[\Delta Q_{1}-(1-K \tau) \Delta Q_{2}\right] d t
$$

When we observe the triangle SMN in figs. 5c and 5d, it is possible to observe the following dependence:

$$
\begin{aligned}
& k=\operatorname{tg} \alpha=\frac{d h}{d Q_{2}} \\
& K^{*}=\frac{1}{k}=\frac{1}{\operatorname{tg} \alpha}=\operatorname{ctg} \alpha=\frac{d Q_{2}}{d h}
\end{aligned}
$$

$k$ - coefficient of the straight line direction which is a graph of wet land mass sieved through the platform grid.

On the basis of rules known from the theory of differential calculus, we get:

$$
\begin{aligned}
& \frac{d h}{d Q_{2}} \approx \frac{\Delta h}{\Delta Q_{2}} \\
& \Delta Q_{2} \cdot d h \approx \Delta h \cdot d Q_{2} \\
& \Delta Q_{2} \approx \frac{d Q_{2}}{d h} \Delta h \approx \operatorname{ctg} \alpha \cdot \Delta h \approx K^{*} \cdot \Delta h
\end{aligned}
$$

By substituting relation (7) into (4), the increase of the level of wet land mass $\Delta h$ will be, [2]:

$$
\begin{aligned}
& d(\Delta h)=\frac{1}{P}\left[\Delta Q_{1}-(1-K \tau) \cdot K^{*} \cdot \Delta h\right] d t \quad / \cdot \frac{P}{d t} \\
& P \frac{d(\Delta h)}{d t}=\Delta Q_{1}-K^{*} \cdot \Delta h+K_{\tau} \cdot K^{*} \cdot \Delta h
\end{aligned}
$$

$$
\begin{aligned}
& P \frac{d(\Delta h)}{d t}=\Delta Q_{1}-\left(K^{*}-K_{\tau} \cdot K^{*}\right) \Delta h \\
& P \frac{d(\Delta h)}{d t}+\left(K^{*}-K_{\tau} \cdot K^{*}\right) \Delta h=\Delta Q_{1} \\
& P \frac{d(\Delta h)}{d t}+K^{*}\left(1-K_{\tau}\right) \Delta h=\Delta Q_{1} \quad /: K^{*} \\
& \frac{P}{K^{*}} \frac{d(\Delta h)}{d t}+\left(1-K_{\tau}\right) \Delta h=\frac{1}{K^{*}} \Delta Q_{1}
\end{aligned}
$$

Taking into account the equation (6), the following expression can be written:

$$
k \cdot P \frac{d(\Delta h)}{d t}+\left(1-K_{\tau}\right) \Delta h=k \cdot \Delta Q_{1}
$$

Increased level of wet land mass on a platform $\Delta h$ according to (3) is clearly a function of both $\Delta Q_{1}$ and $\Delta Q_{2}$. However, since the value of $\Delta Q_{2}$ is inserted into mathematical model through equation (7), it does not exist directly in equation (8); instead, it exists indirectly through the coefficient of delay of sieved land mass relative to the delivered land mass $K_{\tau}$. Therefore, equation (8) clearly shows the functional relationship between the increased level of wet land mass on the platform $\Delta h$ and the increase of delivery of wet land mass on the platform $\Delta Q_{1}$ :

$$
\Delta h=f\left(\Delta Q_{1}\right)
$$

Viewed from a mathematical point of view, the former dependency is expressed as follows:

$$
y=f(x)
$$

Taking into account the relations (8), (9) and $\left(9^{\prime}\right)$ it is possible to form following identical equalities:

$$
k \cdot P \equiv T_{V} ; k \equiv \frac{1}{K^{*}} \equiv K_{P} ; \Delta h \equiv y ; \Delta Q_{l} \equiv x
$$

Substituting identical relation (10) into relation (8) and bearing in mind the fact that all real physical systems operate in real time $t$, we get the following relationship between previously mentioned values:

$$
T_{V} \frac{d y(t)}{d t}+\left(1-K_{\tau}\right) y(t)=K_{P} \cdot x(t-\tau)
$$

where:

$T_{V}$ - time (inertial) constant of the system E-P-WP (environment-platform-working parameters);

$K_{P}$ - coefficient (factor) of amplification of the system E-P-WP (environment-platform-working parameters);

$\tau$ - time delay of the output signal of the system E-P-WP (environment-platform-working parameters) with respect to the input signal.

The differential equation (11) is a first order differential equation and presents a mathematical model of the process of separation of wet land mass by using oscillatory transport platform in the function of time $t$. 


\section{BASIC CHARACTERISTICS OF THE SYSTEM E-P-WP (ENVIRONMENT-PLATFORM-WORKING PARAMETERS)}

General solution of the differential equation (11) represents the system (platform) response to the input excitation, whereas two mutually different states of the system are obvious [8]:

$>$ nonstationary transitional state of the system - it is a part of the response approaching zero as an independent variable, i.e. time tends to infinity $(t \rightarrow \infty)$, and it is characterized by the so-called "response time of the platform" $\left(t_{o p}\right)$. This response time is the speed of calming platform due to input disturbance, i.e. it represents the length of the transition process;

$>$ stationary state of the system - it is a part of the response that, after the completion of the transition process, instead of tending to zero when the independent variable, i.e. time tends to infinity $(t \rightarrow \infty)$, it approaches the previously defined constant value $y(t)=$ const, which in the theory of the systems is also called the signal of the zero frequency (DC signal). DC signal, i.e. stationary response of the platform that separates wet land mass can be determined on the basis of the theory of limiting value:

$$
f(\propto)=\lim _{t \rightarrow \infty} f(t)=\lim _{p \rightarrow 0} p \cdot F(p)
$$

$p$ - complex variable which helps to derivate Laplace transform and inverse Laplace transform.

It is particularly important to emphasize the fact that the response time of the platform $t_{o p}$ which presents the calming speed of the disturbed motion does not depend on the type of disorder, but only on the design parameters of the platform. Depending on the duration of the platform calming after any intentionally or accidentally induced disorder, there are two typical cases in practice [4]:

$$
\begin{aligned}
& \rightarrow \text { case I: if } t_{\mathrm{op}}>t_{\mathrm{rp}} \rightarrow \begin{array}{l}
\text { correction by the open loop } \\
\text { system; }
\end{array} \\
& \rightarrow \text { case II: if } \mathrm{t}_{\mathrm{op}}<\mathrm{t}_{\mathrm{rp}} \rightarrow \begin{array}{l}
\text { correction by the closed } \\
\text { loop feedback; }
\end{array}
\end{aligned}
$$

$t_{r p}$ - time of platform reaction (minimum time required to change the platform mode by changing one or more operating parameters).

Effects of different disturbance factors in the environment (disturbing forces and moments, unfavorable composition of the soil, high humidity, etc.) cause the difference between the input, i.e. the amount of wet land mass delivered onto the platform $\left(Q_{l}\right)$ and output, i.e. the actual amount of wet land mass sieved through the platform grid $\left(Q_{2}\right)$. The resulting difference is necessary to somehow correct.

In the first case, if the platform response time $t_{o p}$ is relatively long (slow calming after the disturbance) and if it is longer than the platform reaction time $t_{r p}$, the platform cannot receive the output signal and it will perform control operations on a predetermined program. This is a correction by the open loop system where the correction itself may present a new disorder, which makes the sieving of wet land mass even more difficult (Fig. 6).

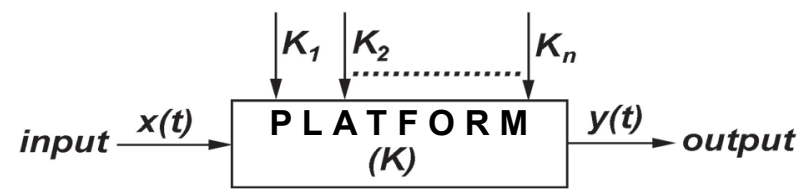

Fig 6. Structure scheme of an open system E-P-WP (environment-platform-working parameters)

Symbols in figure 6 have the following meanings:

$x(t)$ - input which replaces the effect of environment on the observed system (independent variable);

$y(t)$ - output which replaces the effect of the system on the environment (dependent variable);

$K$ - transformation operator which contains all elementary operations $\left(K_{l}, K_{2}, \ldots, K_{n}\right)$ being performed on the input $x(t)$ to obtain the output $y(t)$.

In another case, if the response time of the platform $t_{o p}$ is relatively short (fast calming after the disturbance) and if it is shorter than the platform reaction time $t_{r p}$, it is possible, through a feedback, to transmit the output signal to control system of the platform, so that the sieving of wet land mass can achieve the desired (predetermined) value by changing the platform mode. This is a typical correction by closed-loop feedback (Fig. 7).

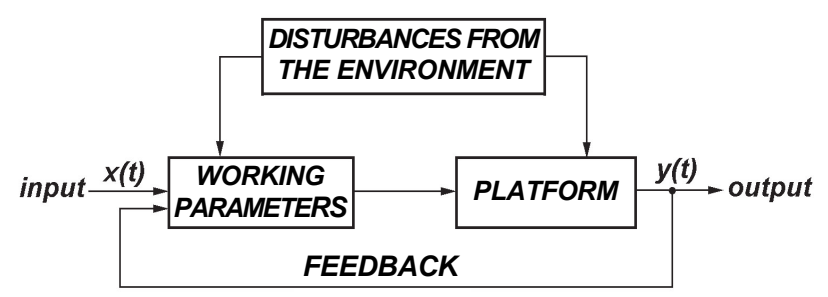

Fig. 7. Structure scheme of the closed system E-P-WP (environment-platform-working parameters)

Therefore, the following conclusions can be made [5]:

$>$ in all types of disturbances (either from the environment or due to the reaction of the platform itself) it is necessary to calm the performance of the platform as quickly as possible so that it tends to a steady operating mode;

$>$ it is desirable to make the response time of the platform as well as its reaction time as short as possible, in order to achieve greater safety and reliability in operation, i.e. so that the platform can follow all new circumstances in real time;

$>$ after calming, that is after the completion of the transition process, the platform should be at operating mode that is close to the desired operating mode of sieving wet land mass.

If all three requirements are met, the work of oscillatory transport platform is said to operate in an expected manner, i.e. the choice of working parameters 
provides a stable platform operation from the aspect of separating and sieving of wet land mass.

\section{ANALYSIS OF THE DYNAMIC BEHAVIOR OF OSCILLATORY TRANSPORT PLATFORM DURING SEPARATING AND SIEVING WET LAND MASS}

Dynamic behavior of the platform that separates and sieves wet land mass is identified by the method of active experiment, i.e. method of system response to a known excitation (test) function. The input used in this method is a known disturbance function, and the behavior of the entire system is concluded on the basis of the response, i.e. the output of the system.

There is a relatively large number of disturbance test functions, i.e. applicative inputs that are applied in practice [8]. For the studied case of sieving wet land mass, the excitation caused by the action of jumping function $x_{0}(t)$ at the system input is the most appropriate to observe. The excitation by means of harmonic function $F_{0} \sin (\Omega t)$ is analyzed within the method of frequency response of the system E-P-WP.

\subsection{Testing of system E-P-WP by jumping function $x_{0}(t)$}

For this analysis, first it is necessary to solve the differential equation (11), the obtained solution representing response of the studied system to excitation jumping function $x_{0}(t)$. In general, the general solution of linear differential equation with constant coefficients consists of two parts:

$\rightarrow$ homogeneous solution $\boldsymbol{y}_{\boldsymbol{h}}(\boldsymbol{t})$ which depends only on the structural and exploitation characteristics of the system (platform), and it does not depend on the type of the applied input signal $x(t)$. Thus this solution in terms of control theory is the free response of the system.

$\rightarrow$ particular solution $y_{p}(t)$ which depends on the type of the applied input signal $x(t)$ and which, together with all its coefficients satisfies the starting equation, i.e. the mathematical model. From the aspect of control theory this solution is the forced response of the system.

The input excitation signal, i.e. jumping function $x_{0}(t)$ acting at the entrance of E-P-WP system is defined by the following expression (fig. 8.a):

$$
x(t)=\left\{\begin{array}{c}
0 \text { за } t<0 \\
x_{0} \text { з } а \text { t } t \geq 0
\end{array}\right.
$$

Action of the input signal results in the output signal $y(t)$ which is delayed relative to the input signal for the value of the time delay $\tau$, wherein the output signal is defined by the expression (fig. 8.b):

$$
\begin{aligned}
& y(t)=\left\{\begin{array}{c}
0 \text { за } t<\tau \\
h(t) \text { за } t \geq \tau
\end{array}\right. \\
& h(t) \text { - function of transitional characteristic. }
\end{aligned}
$$

Homogeneous solution is obtained as a solution that satisfies the left side of the differential equation (11) when equaled to zero:

$$
y_{h}(t)=A e^{\left(\frac{K_{\tau}-1}{T_{V}}\right) t}
$$

$A$ - constant of the system, which is determined from defined initial conditions.

Particular solution depends on the type of input signal applied and its value for given input signal $x_{0}(t)$ is:

$$
y_{p}(t)=\frac{K_{P} \cdot x_{0}(t-\tau)}{1-K_{\tau}}
$$

General solution of differential equation representing the mathematical model of the system under study is obtained as the sum of the homogeneous and particular solutions:

$$
y(t)=A e^{\left(\frac{K_{\tau}-1}{T_{V}}\right) t}+\frac{K_{P} \cdot x_{0}(t-\tau)}{1-K_{\tau}}
$$

For the sake of illustration, the graphical interpretation of all above considerations is given in Figure 8 . The figure shows that the output signal $y(t)$ begins to generate after a certain time $\tau$ representing the time of its delay relative to the input signal. Therefore, the studied system E-P-WP can be said to behave like a typical first-order aperiodic element with delay, whose characteristics and dynamic behavior are well-known from the theory of linear systems.
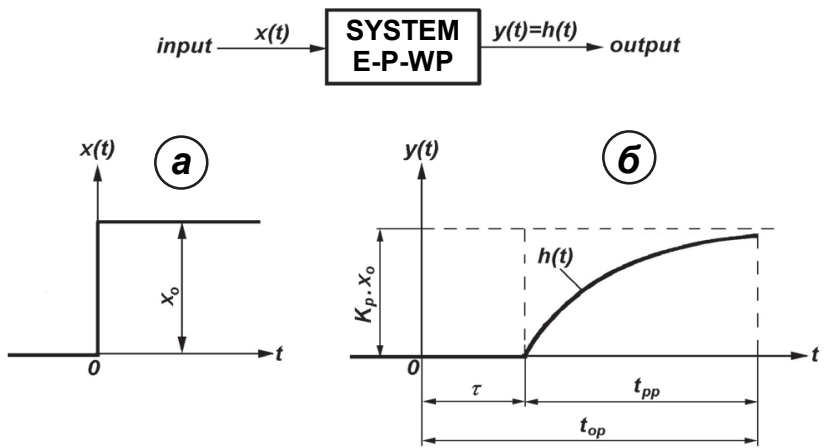

Fig. 8. Response of oscillatory transport platform separating wet land mass to disorder caused by jumping function ( $\tau$-delay time of the output signal relative to the input signal; $t_{p p}$-length of transition process; $t_{\text {op-response time of the platform) }}$

The figure also shows that jumping response $y(t)$ of the platform separating wet land mass does not achieve currently defined value $K_{P} \cdot x_{0}$ after the expiry of the time delay $\tau$; it gradually approaches the value asymptotically. Theoretically, defined value $K_{P} \cdot x_{0}$ would be achieved only after infinite time, which indicates that the platform has a certain imperfection, or inertia, the measure of the inertia being the time constant $T_{V}$. The greater the time constant, the greater the inertia of the platform during the process of separation [6].

It is believed that the transition process is completed when the function of the transitional characteristic $h(t)$ reaches the value $h(t)=y(t) \approx 0,95 \cdot K_{P} \cdot x_{0}$ (approximately 
$t \approx 3 \cdot T_{V}$ ), because a new balance of the system E-P-WP is then established.

\subsection{Transmission function of the system E-P-WP}

The transmission function is one of the most significant features of every system being studied, for it may be the basis of many important conclusions on dynamic characteristics and stability of the entire system during its performance. Also, a complete frequency analysis of the system observed can be based on it.

The transmission function $G(p)$ is defined as the relation between L-transformation of response and Ltran-sformation of excitation of the system, [8]:

$$
G(p)=\frac{L-\text { transformation of response }}{L \text {-transformation of stimulus }}=\frac{Y(p)}{X(p)}
$$

with two basic assumptions: are nonexistent;

$\rightarrow$ for $\boldsymbol{t}<\mathbf{0}$, input signal $x(t)$ and output signal $y(t)$

$\rightarrow$ for $\boldsymbol{t}=\mathbf{0}$, all initial conditions are equal to zero.

Transmission function of the system E-P-WP is obtained by making Laplace transform of the left and right sides of the equation (11) which represents the mathematical model of the system under consideration:

$$
L\left[T_{V} \frac{d y(t)}{d t}+\left(1-K_{\tau}\right) y(t)\right]=L\left[K_{P} \cdot x(t-\tau)\right],
$$

from which we conclude:

$$
G(p)=\frac{K_{P}}{T_{V} \cdot p+1-K_{\tau}} e^{-\tau p}
$$

$e^{-\tau p}-$ a delay operator the output signal relative to the input signal (due to the sticking of wet land mass to platform grid).

In general case, transmission function of the system can be presented as a fraction, with the necessary condition that numerator and denominator have no common solutions:

$$
G(p)=\frac{M(p)}{N(p)}
$$

$M(p)$ - polynomial of " $p$ " of numerator;

$N(p)$ - polynomial of " $p$ " of denominator.

This form of presenting transmission function allows relatively fast determination of its zeros and poles, with the aim of its graphical representation in the plane of the complex variable $p=c+j \omega$.

\section{- Zeros of the transmission function $G(p)$ :}

When the numerator in (21) equals zero, we get:

$$
G(p)=0 \text {, for } M(p)=0 \text {. }
$$

In this case, the points $z_{i}(i=1,2, \ldots, n)$, where the transmission function $G(p)$ has the zero value, are zeroes of transmission function. In the case of the transmission function (20) we obtain:

$$
G(p)=0 \text {, за } K_{P} \cdot e^{-\tau p}=0 \Leftrightarrow K_{P}=0 \vee e^{-\tau p}=0
$$

But, since amplification coefficient of the system $K_{P}$ and delay operator $e^{-\tau p}$ are different from zero, it can be concluded that condition $G(p)=0$ can never be satisfied, which implies that transmission function of the system E-P-WP has no zeroes in the plane of complex variable $p=c+j \omega$.

\section{- Poles of the transmission function $G(p)$ :}

When denominator in (21) equals zero and is solved by " $p$ " we get:

$$
N(p)=0
$$

In this case, the points $p_{i}(i=1,2, \ldots, n)$, where denominator has the zero value, represent poles of transmission function. In the case of the transmission function (20) we obtain:

$$
\begin{aligned}
& N(p)=T_{V} \cdot p+1-K_{\tau}=0 \\
& T_{V} \cdot p=K_{\tau}-1 \\
& p=\frac{K_{\tau}-1}{T_{V}}
\end{aligned}
$$

The above mentioned statement, that $K_{\tau}=1, p=0$, is possible in terms of mathematics (not in terms of physics), holds true for this case as well, so it is not taken into consideration.

Delay operator contained in (20) has the following value in the case of analyzed system E-P-WP:

$$
e^{-\tau p}=e^{-\tau\left(\frac{K_{\tau}-1}{T_{V}}\right)}=e^{\left(\frac{1-K_{\tau}}{T_{V}}\right) \tau}
$$

\section{- "PZ-diagram" of E-P-WP system:}

Both zeros and poles of the transmission function $G(p)$ can be presented graphically on the same chart in the plane of complex variable $p=c+j \omega$. Thus pole-zero diagram enables a good overview and much easier analysis of dynamic behaviour of any individual system. 


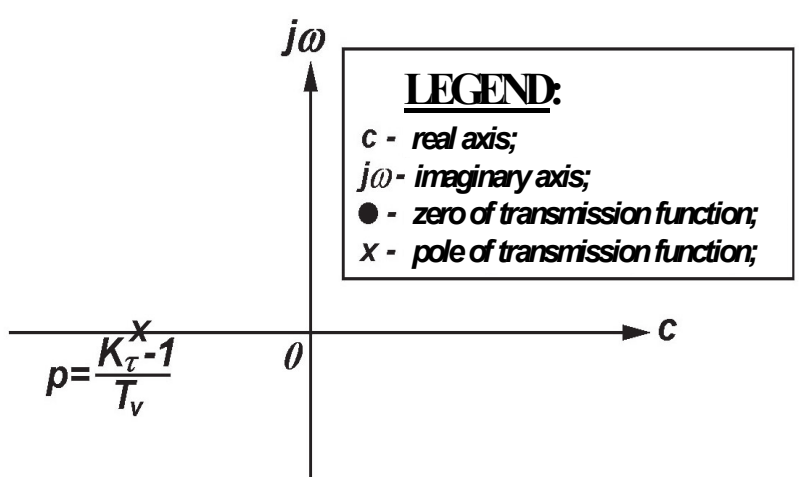

Fig. 9. PZ-diagram of oscillatory transport platform that performs the separation of wet land mass

\subsection{Testing E-P-WP system by the method of frequency response of the system}

If $c=0$ in the complex variable $p=c+j \omega$, we get a complex frequency $p=j \omega$, so in this case it is possible to analyze the system in the frequency domain instead of domain of complex variable by the process known as THE METHOD OF FREQUENCY RESPONSE. Frequency response is the stationary response of the system to harmonic excitation function (sine or cosine harmonic test function).

This type of excitation is distinguished by the input harmonic signal which causes the output harmonic signal with the frequency of the input signal (forced oscillations) and with the amplitude and phase which are significantly different from the amplitude and phase of the input signal. Mathematical interpretation is shown in Fig. 10.
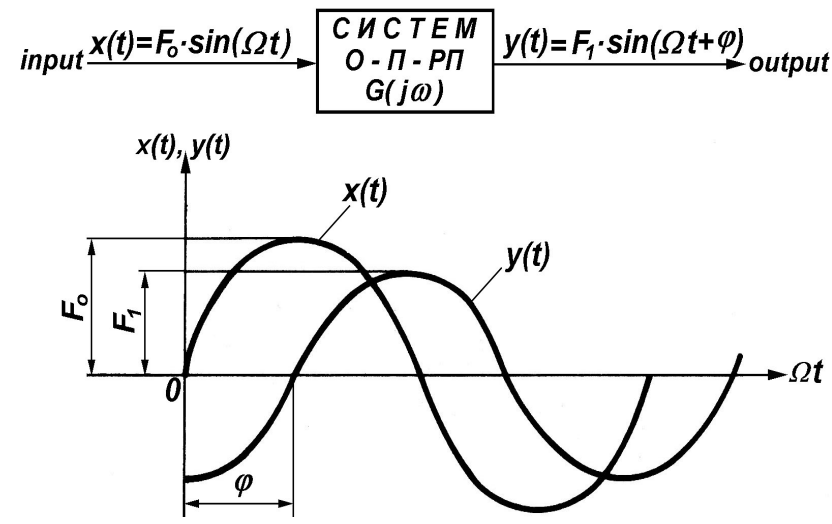

Fig. 10. Response of oscillatory transport platform that performs the separation of wet land mass to disturbance caused by harmonic excitation function

Input and output harmonic signals can be shown as follows [8]:

$$
\begin{aligned}
\rightarrow & \text { input harmonic signal: } \\
& x(t)=F_{0} \cdot \sin (\Omega t) \\
\rightarrow & \text { output harmonic signal: } \\
& y(t)=F_{1} \cdot \sin (\Omega t+\varphi)
\end{aligned}
$$

$F_{0}$ - oscillation amplitude of the input signal;

$F_{l}$ - oscillation amplitude of the output signal;
$\Omega$ - angular frequency of oscillation of external disturbing force.

If the domain of complex variable $p=c+j \omega$ moves into the domain of complex frequency $p=j \omega$ (by $c=0)$, we obtain the frequency characteristic $G(j \omega)$ based on equations (20) and (23). It represents transmission function of the system during the harmonic (sinusoidal) excitation:

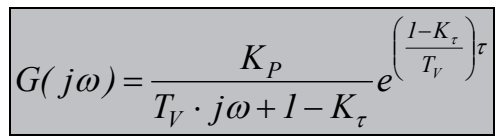

If the frequency of oscillation $\Omega$ of input signal $x(t)$ is changed while maintaining the amplitude of oscillation at a constant level $\left(F_{0}=\right.$ const $)$, the output signal $y(t)$ is obtained which has precisely determined value of amplitude $F_{1}$ for every new value of excitation frequency $\Omega$, and has precisely determined value of phase displacement $\varphi$ in relation to the input signal:

$$
\begin{aligned}
& F_{l}=F_{l}(\Omega) ; \varphi=\varphi(\Omega) \\
& \frac{F_{1}}{F_{0}}=M(\Omega)
\end{aligned}
$$

$M(\Omega)$ - amplification factor of the system E-P-WP (its dependence on the relationship between amplitudes of output and input harmonic signals is the amplitude-frequency characteristic of the system);

$\varphi(\Omega)$ - phase displacement of the output signal relative to the input harmonic signal (dependence of the displacement on $\Omega$ represents the phase-frequency characteristic of the system).

\section{- Amplitude-frequency characteristic of the system E-P-WP:}

This characteristic is determined on the basis of frequency characteristic (26) as the absolute value of the frequency characteristic, according to the form:

$$
|G(j \omega)|=M(\omega)=\sqrt{R_{e}^{2}[G(j \omega)]+I_{m}^{2}[G(j \omega)]}
$$

On the basis of equations (26) and (29) we obtain:

$$
\begin{aligned}
& R_{e}[G(j \omega)]=\frac{K_{P} \cdot e^{\left(\frac{1-K \tau}{T_{V}}\right) \tau} \cdot\left(1-K_{\tau}\right)}{\left(1-K_{\tau}\right)^{2}+T_{V}^{2} \cdot \omega^{2}} \\
& I_{m}[G(j \omega)]=-\frac{K_{P} \cdot e^{\left(\frac{1-K \tau}{T_{V}}\right) \tau} \cdot T_{V} \cdot \omega}{\left(1-K_{\tau}\right)^{2}+T_{V}^{2} \cdot \omega^{2}}
\end{aligned}
$$

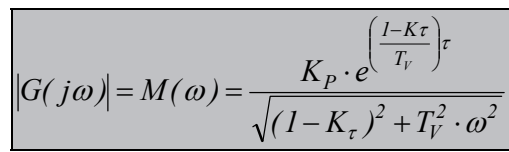

Equation (32) represents the amplitude-frequency characteristic, i.e. amplification factor of the E-P-WP system.

- Phase-frequency characteristic of the system E-P-WP: 
Phase-frequency characteristic is also determined on the basis of frequency characteristic $G(j \omega)$, i.e. by its real and imaginary part:

$$
\begin{aligned}
& \operatorname{tg} \varphi=\frac{I_{m}[G(j \omega)]}{R_{e}[G(j \omega)]}=-\frac{T_{V} \cdot \omega}{1-K_{\tau}} \\
& \varphi=\operatorname{arctg}\left(-\frac{T_{V} \cdot \omega}{1-K_{\tau}}\right)
\end{aligned}
$$

Equation (33) represents the phase-frequency characteristic, i.e. delay characteristic of the output harmonic signal relative to the input harmonic signal of the E-P-WP system.

\section{- Polar diagram of frequency characteristic of the system} E-P-WP:

If the real values of frequency characteristic for various values of frequency $\omega$ are calculated according to equation (30) and if they are presented at the real axis of polar coordinate system, while imaginary values calculated according to equation (31) are presented at imaginary axis, we get the coordinates of the points which, when combined with each other, form a curve known as POLAR DIAGRAM OF FREQUENCY CHARACTERISTIC. This diagram illustrates the results of a complete analysis of the system using the method of frequency response, so it is also as amplitude-phase frequency characteristic of the system.

Table 1. Amplitude-phase frequency characteristic of the system E-P-WP for different values of the excitation frequencies $\omega$

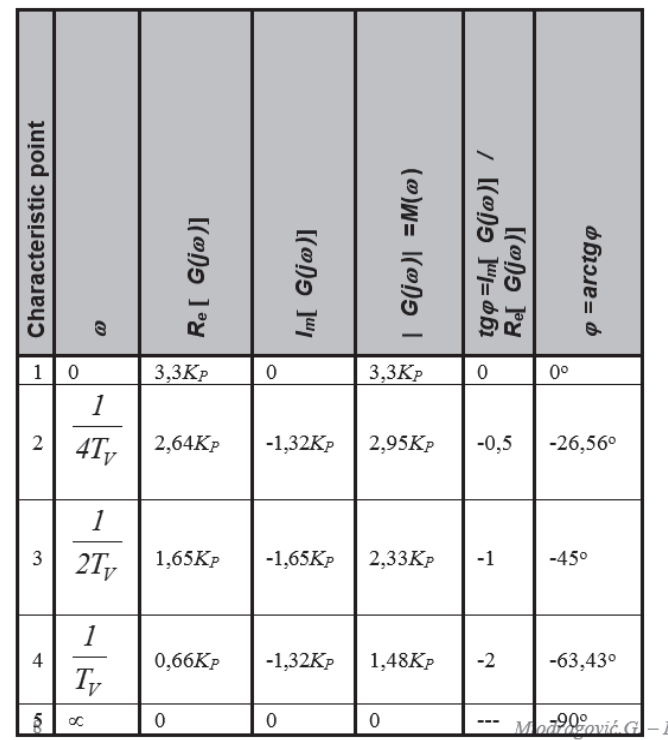

11 relevant data needed to draw polar diagram can be presented in tabular form (see Table 1) for five characteristic points, i.e. characteristic values of excitation frequencies $\omega$. The following assumptions are made for easier and simpler drawing of the diagram [2]: $t_{o p}=3 T_{V}-$ response time of the platform (including time of delay $t$ );

$\tau=T_{V}$ - delay time is equal to the temporal and inertial constant of the system;

$K_{\tau}=0,5$ - delay coefficient of sieved land relative to delivered land (because in reality it is within the range $K_{\tau}=\left[\begin{array}{lll}0 & \ldots & 1\end{array}\right]$ );

$$
e^{\left(\frac{1-K_{\tau}}{T_{V}}\right) \tau}=e^{1-K_{\tau}}=e^{1-0,5} \approx 1,65 \text { - operator }
$$
delay of the E-P-WP system.

The polar diagram of the frequency characteristic $G(j \omega)$ can be drawn based on Table 1, as shown in Figure 11.

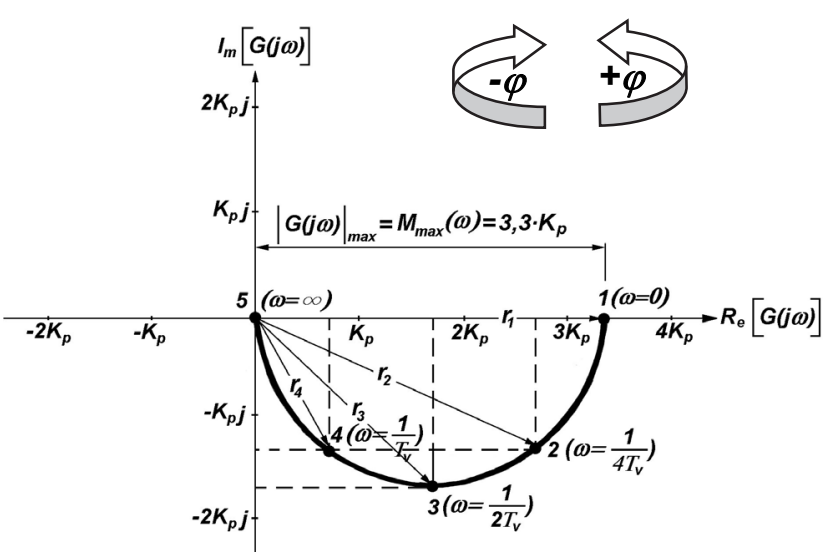

Fig. 11. Polar diagram of the frequency characteristic $G(j \omega)$ of oscillatory transport platform that performs the separation of wet land mass

$\rightarrow$ The polar diagram shows that the system E-P-WP has maximum amplification (ratio of the amplitudes of the output and the input harmonic signals) of $3,3 K_{P}$ for $\omega=0$, when there is no phase displacement between the input and output harmonic signals $\left(\varphi=0^{\circ}\right)$.

$\rightarrow$ If frequency $\omega$ increases, of amplification of the system decreases continuously, and it equals zero for $\omega \rightarrow \infty$. Then the phase displacement between the input and output harmonic signals is maximum and it is $\varphi(\propto)=-(\pi / 2) \mathrm{rad}=-90^{\circ}$, which corresponds to one quarter of the oscillation period.

$\rightarrow$ If $\tau$ and $K_{\tau}$ have other values, operator delay of the system E-P-WP will be different and the coordinates of characteristic points will be different but the basic look of the polar diagram remain unchanged, i.e. it will always be identical to the diagram shown in Figure 11.

\section{STABILITY OF OSCILLATORY TRANSPORT PLATFORM DURING SEPARATION AND SIEVING OF WET LAND MASS}

The general stability of the mechanical system is the ability to, after the cessation of any disturbance, retake its desired position (definition according to Lyapunov). This means that: if the platform is moved from its stable 
position by any outside or any internal disturbance and if it quickly returns to the reference position upon the cessation of these disturbances, then its work is STABLE.

Otherwise, if the difference between the parameters $Q_{l}$ and $Q_{2}$ becomes greater after cessation of disturbance, then the work of platform is UNSTABLE because of large deviation from the expected difference between delevered and sieved quantity of wet land mass.

\subsection{Stability of the system E-P-WP according to the Routh criterion}

Routh criterion for testing system stability is based on the analysis of characteristic equation $N(p)$ of transmission function $G(p)$ :

$$
N(p)=a_{n} \cdot p^{n}+a_{n-1} \cdot p^{n-1}+\ldots+a_{1} \cdot p+a_{0}=0
$$

Routh table is based on the characteristic equations according to the following scheme:

$$
\begin{array}{c|cccc}
p^{n} & a_{n} & a_{n-2} & a_{n-4} & \ldots \\
a_{n-1} & a_{n-3} & a_{n-5} & \ldots \\
b_{1} & b_{2} & b_{3} & \ldots \\
c_{1} & c_{2} & \cdots & \\
d_{1} & \ldots & & \\
\cdots & & &
\end{array}
$$

of Routh formulas).

$b_{i}, c_{i}, d_{i}-$ Routh coefficients (calculated by means In the case of E-P-WP system, characteristic equation and Routh table have the following form:

$$
\begin{aligned}
& N(p)=T_{V} \cdot p+1-K_{\tau}=0 \\
& \begin{array}{c|cc}
\cline { 2 - 3 } p^{l} & T_{V} & 0 \\
p^{0} & 1-K_{\tau} & 0
\end{array}
\end{aligned}
$$

Routh stability criterion states that:

"The system is stable only when all the elements of the first column of Routh table have the same sign" (this is both necessary and sufficient condition for stability).

Since $T_{V}>0$ and $\left(1-K_{\tau}\right)>0$ (both elements in the first column of the Routh table are always positive, i.e. they have the same sign), the system E-P-WP is stable according to Routh criterion.

\subsection{Stability of E-P-WP system according to Hurwitz criterion}

This criterion is also based on the analysis of the characteristic equation of the system $N(p)$ of transmission function $G(p)$ :

$$
N(p)=a_{n} \cdot p^{n}+a_{n-1} \cdot p^{n-1}+\ldots+a_{1} \cdot p+a_{0}=0
$$

The chief determinant of system $\Delta_{n}$ (so-called Hurwitz determinant) is based on the characteristic equation according to the following general scheme:

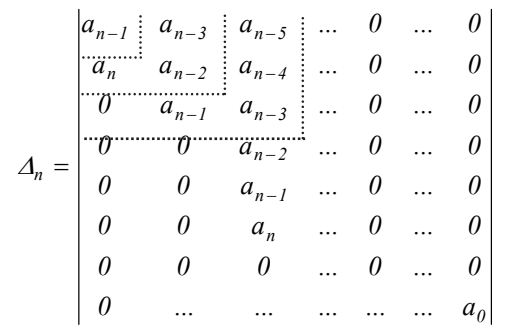

Subdeterminants are:

$$
\Delta_{1}=a_{n-1} ; \quad \Delta_{2}=\left|\begin{array}{cc}
a_{n-1} & a_{n-3} \\
a_{n} & a_{n-2}
\end{array}\right| ; \quad \Delta_{3}=\left|\begin{array}{ccc}
a_{n-1} & a_{n-3} & a_{n-5} \\
a_{n} & a_{n-2} & a_{n-4} \\
0 & a_{n-1} & a_{n-3}
\end{array}\right|
$$

In the case of EE-P-WP system, characteristic equation and chief Hurwitz's determinant have the following form (in this case there are no subdeterminants because the characteristic equation is the first degree equation):

$$
\begin{aligned}
& N(p)=T_{V} \cdot p+1-K_{\tau}=0 \\
& \Delta_{n}=\Delta_{l}=\left|a_{n-1}\right|=\left|1-K_{\tau}\right|>0
\end{aligned}
$$

Hurwitz criterion of stability states that:

"The system is stable if both main determinant of the system and all its subdeterminants are positive at the same time, with necessary precondition that the coefficient in the first element of the characteristic equation is positive, too $\left(a_{n}>0\right)^{\prime \prime}$.

Since the main determinant is $\Delta_{l}>0$, there are no subdeterminants and $\left(a_{n}=T_{V}\right)>0$, the system E-P-WP is STABLE according to Hurwitz criterion, too.

\section{RESERVE OF STABILITY OF OSCILLATORY TRANSPORT PLATFORM DURING SEPARATING AND SIEVING WET LAND MASS}

In this case, first we have to determine the value of maximum amplification of the system $\boldsymbol{M}_{\max }(\boldsymbol{\omega})$ for the case of resonance, when the external excitation frequency is equal to the natural frequency of the system. For this purpose, it is necessary to introduce a substitution $\omega=\omega_{0}$ in equation (32) to obtain [2]:

$$
|G(j \omega)|_{\max }=M_{\max }(\omega)=\frac{K_{P} \cdot e^{\left(\frac{1-K_{\tau}}{T_{V}}\right) \tau}}{\sqrt{\left(1-K_{\tau}\right)^{2}+T_{V}^{2} \cdot \omega_{0}^{2}}}
$$

The definition states that relative stability, i.e. STABILITY RESERVE of E-P-WP system is inversely proportional to $M_{\max }(\omega)$ :

$$
R_{S}=\frac{1}{M_{\max }(\omega)}=\frac{\sqrt{\left(1-K_{\tau}\right)^{2}+T_{V}^{2} \cdot \omega_{0}^{2}}}{K_{P} \cdot e^{\left(\frac{1-K_{\tau}}{T_{V}}\right) \tau}}
$$

On the basis of (44) it can be concluded that reserve stability of oscillatory transport platform during separating and sieving wet land mass is greater if:

$\rightarrow$ inertia of the platform is greater (expressed through time constant $T_{V}$ ); 

is greater;

$\rightarrow$ natural frequency $\left(\omega_{0}\right)$ of platform oscillation

$\rightarrow$ amplification factor $\left(K_{P}\right)$ of the platform is smaller; $\rightarrow$ operator delay of the platform $e^{\left(\frac{1-K_{\tau}}{T_{V}}\right) \tau}$ is smaller, i.e. delay time $\tau$ is shorter.

If reserve of stability of platform $R_{S}$ is greater there is less possibility to disturb the process of separation and sieving of wet land mass during resonance and frequencies that are close to the resonant ones $\left(\omega \approx \omega_{0}\right)$. In some extreme cases, plaform productivity may fall below a minimum technical and economic criterion, or the platform may even stop working.

\section{CONCLUSION}

This paper presents the research results of the process of separation and sieving of wet land mass which is done by vibrating oscillatory transport platform which, at the same time, should enable optimum transport of wet land mass. All relevant results are based on dynamic analysis of said processes within the scope of theoretical phase of researching the problems mentioned above.

- Equation (11) is a first order differential equation representing the mathematical model of the process of separation and sieving of wet land mass by oscillatory transport platform in real exploitation conditions and in real time $(t)$.

- The mathematical model (11) and transmission function (20) are the basis for analysis of dynamic behaviour of E-P-WP system. Due to the fact that the transmission function $G(p)$ does not possess a pole or a zero in the coordinate beginning of the complex plane " $p$ ", and since it contains time delay $\tau$, the E-P-WP system is a specific combination that, in the theory of modelling, is known as the first order aperiodic element with time delay. "PZ-diagram" of this element is shown in Figure 9.

- The amplitude-frequency characteristic of system (32) and phase-frequency characteristic of the system (33) are the basis for drawing a polar diagram of frequency characteristic $G(j \omega)$ which is shown in Fig. 11. This diagram shows the dependence between the quantity of sieved wet land mass $Q_{2}$ (output variable) and different oscillation frequencies of external disturbance forces (input variable). Following facts can be stated after analyzing the polar diagram:

* For $\omega=0$ and $\varphi=0^{\circ} \Rightarrow$ amplification of the system is the largest and its absolute value is: $|G(j \omega)|=M(\omega)=3,3 K_{P}$;

* If value $\omega$ is further increased, amplification of the system tends to reduce continuously;

* For $\omega=\propto$ and $\varphi=-\pi r a d=-180^{\circ} \Rightarrow$ amplification of the system disappears (it takes the zero value).

- Based on Routh criterion (37) it is obvious that the process of separation and sieving of wet land mass by oscillatory transport platform is a stable process, for all the elements in the first column of Routh table have the same sign (they have a positive value because of $T_{V}>0$ and $\left(1-K_{\tau}\right)>0$ ). The same conclusion is drawn if Hurwitz stability criterion is applied, because the main determinant is $\Delta_{l}>0$, there are no subdeterminants and the coefficient of the first element of the characteristic equation is $\left(a_{n}=T_{V}\right)>0$.

- On the basis of equation (44), reserve of stability of E-PWP system is greater if the platform inertia (expressed through time constant $T_{V}$ ) and natural frequency of the platform oscillation $\left(\omega_{0}\right)$ are greater, while amplification factor of the platform $\left(K_{P}\right)$ and delay time of the output signal relative to the input signal $(\tau)$ are as small as possible.

\section{REFERENCES}

[1] Mihajlović, G., Utvrđivanje optimalnih radnih parametara vibraciono-njihajucih oscilatornih platformi sa aspekta razbijanja i separacije transportovane mase, M.Sc. thesis, Technical Faculty, Čačak, 1995.

[2] Mihajlović, G., Uticajni parametri na radnu sposobnost oscilatornih transportnih platformi za prosejavanje vlažnih materijala, Ph.D. thesis, Faculty of Mechanical Engineering and construction equipment, Kraljevo, 2014.

[3] Mihajlović, G., Mechanical modelling and dynamical analysis of the process of dry land mass transport by means of vibrating oscillatory platform, IMK-14Istraživanje i razvoj, 18(2012)1, SR, UDK 621 ISSN 0354-6829.

[4] Mihajlović, G., Golubović, D., Razbijanje čvrste mase pomoću vibraciono-njihajuće oscilatorne platforme (original scientific paper), Symposium "Contemporary agriculture" (XXI/98, pages $231 \div 236$ ), Technical Faculty and Agronomic faculty, Čačak, 1998.

[5] Mihajlović, G., Golubović, D., Optimizacija geometrijskih parametara vibraciono-njihajucih oscilatornih platformi, Scientific Conference "Research and development of mechanical components and systems" (pages $595 \div 600$ ), Jahorina, Faculty of Mechanical Engineering, Srpsko Sarajevo, 2002.

[6] Golubović, D., Visokofrekventna-vibraciononjihajuća vadilica krompira tip VWK-10 (prototype), development and research project, Technical Faculty, Čačak, 1994.

[7] Корпенко, Н. А., Сељскохозјајственије машини, Kolos, Moskva, 1973.

[8] Simić, D., Osnovi automatskog upravljanja, Građevinska knjiga, Beograd, 1984.

[9] Tekniken, Focus International Book Produktion, Stockholm, 1984. 